\title{
A fingerprint pair analysis of hERG inhibition data
}

\author{
Clayton Springer ${ }^{*}$ and Katherine L Sokolnicki
}

\begin{abstract}
Background: Drugs that bind to the human Ether-a-go-go Related Gene (hERG) potassium channel and block its ion conduction can lead to Torsade de Pointes (TdP), a fatal ventricular arrhythmia. Thus, compounds are screened for hERG inhibition in the drug development process; those found to be active face a difficult road to approval. Knowing which structural transformations reduce hERG binding would be helpful in the lead optimization phase of drug discovery.
\end{abstract}

Results: To identify such transformations, we carried out a comprehensive analysis of all approximately 33,000 compound pairs in the Novartis internal database which have $I_{50}$ values in the dofetilide displacement assay. Most molecular transformations have only a single example in the data set; however, a few dozen transformations have sufficient numbers for statistical analysis.

Conclusions: We observe that transformations which increased polarity (for example adding an oxygen, or an $s p^{2}$ nitrogen), decreased lipophilicity (removing carbons), or decreased positive charge consistently reduced hERG inhibition between 3- and 10-fold. The largest observed reduction in hERG was from a transformation from imidazole to methyl tetrazole. We also observe that some changes in aromatic ring substituents (for example hydrogen to methoxy) can also reduce hERG binding in vitro.

Keywords: Fingerprint pairs, hERG, Molecular pair, Hydroxyl, Molecular matched pairs, Cliff pairs, Extended connectivity fingerprints (ECFP)

\section{Background}

Inhibition of the human Ether-a-go-go Related Gene (hERG) channel can be a limiting toxicity for drug candidates. The hERG channel regulates transmembrane movement of potassium ions and is a major contributor to the repolarization phase of the cardiomyocyte action potential in the heart [1]. Inhibition of the hERG channel causes lengthening of the cardic QT interval, which can lead to Torsade de Pointes (TdP) [2]. It was this toxicity that in 1997 led to the withdrawal of terfenadine (Seldane) [3]. Although the Redfern criteria is that the $\mathrm{IC}_{50}$ (half maximal inhibitory concentration in vitro) be more than 30 fold greater than the $C_{\max }$ (the maximum plasma concentration in vivo), typically during lead optimization the $\mathrm{C}_{\max }$ (or dose) is not known [2]. However, project teams can estimate toxicity from in vitro $\mathrm{IC}_{50} \mathrm{~S}$. For drugs with submicromolar $C_{\max }$, an in vitro hERG inhibition $\mathrm{IC}_{50}$ of greater than 30 micromolar $(\mu \mathrm{M})$ in the radio-ligand binding assay [4] is generally considered

\footnotetext{
* Correspondence: Clayton.springer@novartis.com

Novartis Institutes for BioMedical Research, 100 Technology Square, Cambridge, MA 02139, USA
}

desirable; having an $\mathrm{IC}_{50}$ of less $10 \mu \mathrm{M}$ is cause for concern and must be improved. The frequency and severity of hERG inhibition drives drug discovery teams to make considerable efforts in measuring, analyzing, and mitigating hERG inhibition [4].

QSAR (Quantitative structure-activity relationship) models using machine learning algorithms $[5,6]$ are established tools for analyzing biological activity data, either with linear (linear discriminate analysis (LDA), partial least squares (PLS) [7]) or non-linear (multi-layer perceptrons [8] support vector machines (SVM), random forest, multivariate adaptive regression splines (MARS) methods. These models find several important descriptors of hERG inhibition, including AlogP or ClogP (measures of lipophilicity), the presence of two lipophilic atoms separated by 10 bonds, fluorine atom count, carbon-carbon double bonds, the presence of a hydroxyl, and partial negative surface area $[7,8]$. Regardless of the method chosen, the resulting mathematical model is smoother than the sharpest changes in the data [9]. This contrasts with the needs of lead optimization, in which one wants 
to find the smallest chemical change that makes the best similar compound. To find the sharpest changes in the data, it is molecular pair analysis rather than global QSAR models that is most applicable approach $[10,11]$.

Matched molecular pairs analysis defines a transformation as a change at an attachment point (this may be generalized to multiple attachment points). Literature reports of analysis of pairs started in the mid-2000s $[9,12,13]$. Leach et al. aggregated their aqueous solubility, plasma protein binding and oral exposure data with pre-specified transformations [14]. In a very comprehensive analysis of GlaxoSmithKlines's internal data, Papadatos et al. analyzed the effects that many matched pairs transformations had on hERG inhibition [15]. The authors analyzed those pairs for context and found several that were statistically different from the overall matched pair average and for hERG they gave details for 3 such examples.

The SALI (Structure-Activity Landscape Index) approach to pairs analysis uses similarity distance (typically fingerprint based) to identify pairs [10]. While SALI may be useful for inspection of individual (also called cliff) pairs, these singleton examples lack statistical significance. To identify transformations that have a Wilcoxon consistent effect on hERG inhibition, we introduce fingerprint pairs, which are an extension of the SALI approach. Aggregating the pairs allows us to make observations about which transformations have an effect on hERG binding (see Figure 1 and Methods section). In contrast with matched pairs approaches, which aggregate the pairs by breaking at a single bond, in our approach the fingerprint pairs implicitly aggregate based on contextual information.

\section{Results and discussion}

With a list of individual fingerprint pairs, we then collect the individual pairs into aggregates. We then make observations about the whole collection of transformations. We move onto the discussion of particular transformations where we have enough supporting examples. We conclude with some observed trends across these pairs.

\section{Aggregate size distribution vs. mean effect of hERG inhibition}

After computing all the transformations in the data, we group the pairs such that those making the same chemical transformation are aggregated together. Each aggregate is summarized by its mean hERG inhibition and the number of pairs in the aggregate (Figure 2). The $x$-axis shows the number of examples it has and the $y$-axis its average change in log (hERG) inhibition. The graph's top and bottom halves are symmetrical about the y-axis because each transformation also appears in reverse. In the reverse transformation, the initial molecule, final molecule, the sign of the difference vector, and the sign of the log (fold change) are all reversed.

To determine which aggregates have sufficient statistical power, we use the Wilcoxon distribution, since we do not assume the data are normally distributed. Most transformations probably have at least a small effect on hERG inhibition; however, for aggregates with 4 or fewer examples (that is almost all aggregates), the Wilcoxon confidence interval includes zero or no change. We discard these aggregates and proceed only with those aggregates containing five or more examples (see Methods for details).

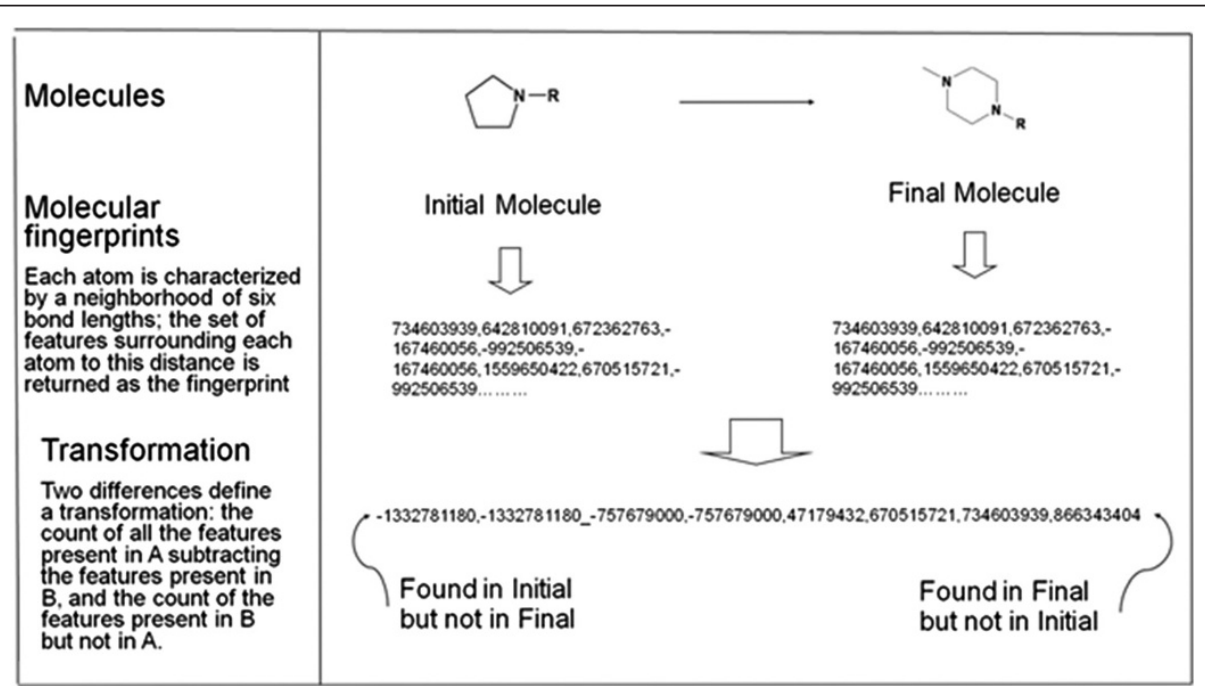

Figure 1 A schematic of a transformation and its ECFP representation. At the top of the schematic, we show the initial and final molecules, and their EFCP fingerprint representation. The last line shows the EFCP representation of the transformation is characterized by which EFCP fingerprints disappear from the initial molecule and which appear in the final molecule. 


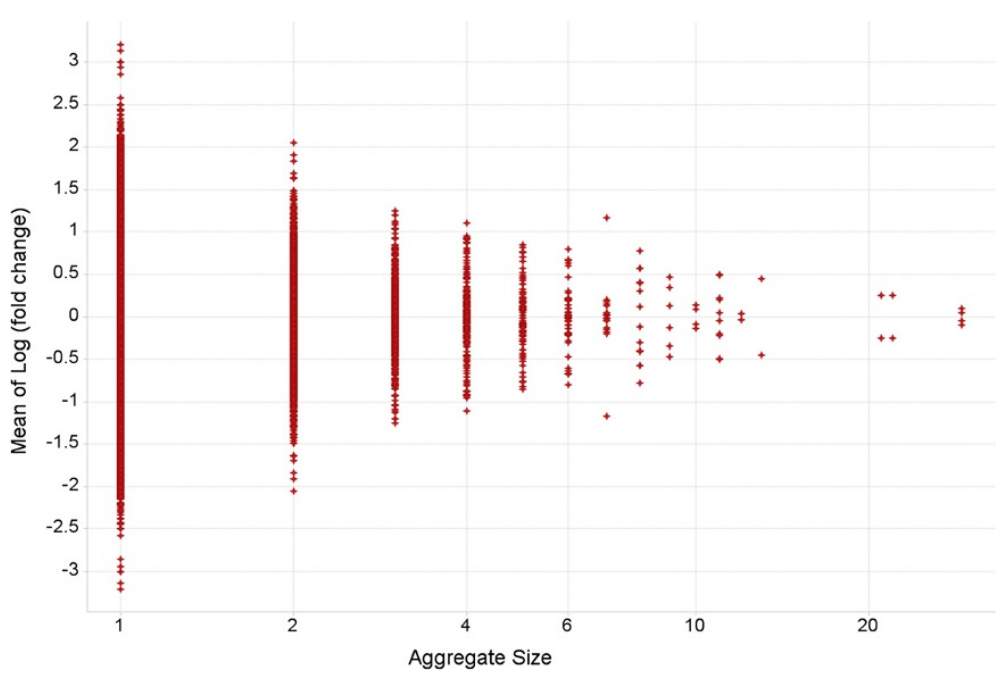

Figure 2 Aggregate size vs. its mean change in log potency. Each point is an aggregate of the same kind of transformation. The x-axis shows the numbers of examples the aggregate has and the $y$-axis shows the geometric average of fold change in each aggregate. See text for discussion.

In our data set of $\sim 33 \mathrm{~K}$ aggregates, there are 112 aggregates with five or more examples; that is, 56 transformations and their reverse transformations. Each is considered individually. While the observed changes in these transformations may be explainable by various models based on SlogP, predicted ionization, and/or aromatic atom counts, such quantitative analysis is beyond the scope of this paper. However, we do note the $\Delta \mathrm{S} \log \mathrm{P}$ in each aggregate.

\section{Size distribution of aggregates follows a power law distribution}

Figure 3 shows the size of aggregates versus the frequency of their occurrence in the data set. These are the examples from the top half of Figure 2 (that is, one direction of the transformation). The distribution of sizes of collected aggregates roughly follows a power law: a few transformations occur commonly, very many occur infrequently, and most transformations are only seen once. The power law distribution is also observed when 3the transformations are aggregated as matched molecular pairs. In this data set, 32,802 of the transformations are singletons (that is, they are size 1); just two aggregates have 28 examples.

\section{Sets of transformations}

As discussed above, only 56 aggregates had sufficient examples to allow for conclusions to be drawn about the chemical transformation. Of these 56, only 17 made a significant reduction in hERG inhibition. Figure 4 through 7 enumerate these transformations. In many of the transformations hERG inhibition follows the expected qualitative trends: decreasing lipophilicity, decreasing basicity, and increasing acidity all decrease the potency of

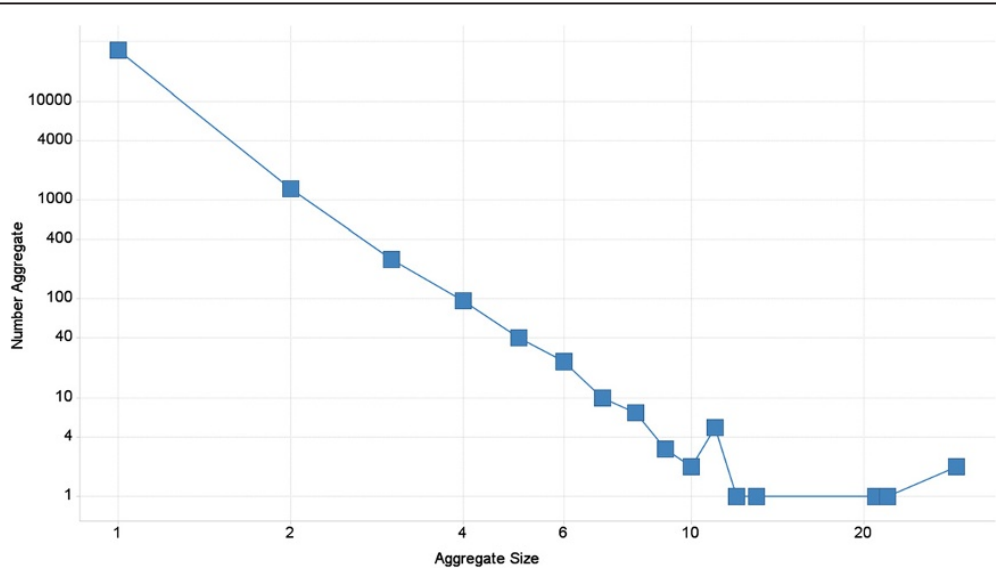

Figure 3 A histogram of aggregate size. The $x$-axis is aggregate size and the $y$-axis is the numbers of aggregates at that size in log scale at that size. Aggregate sizes follows a power law distribution. See text for discussion. 


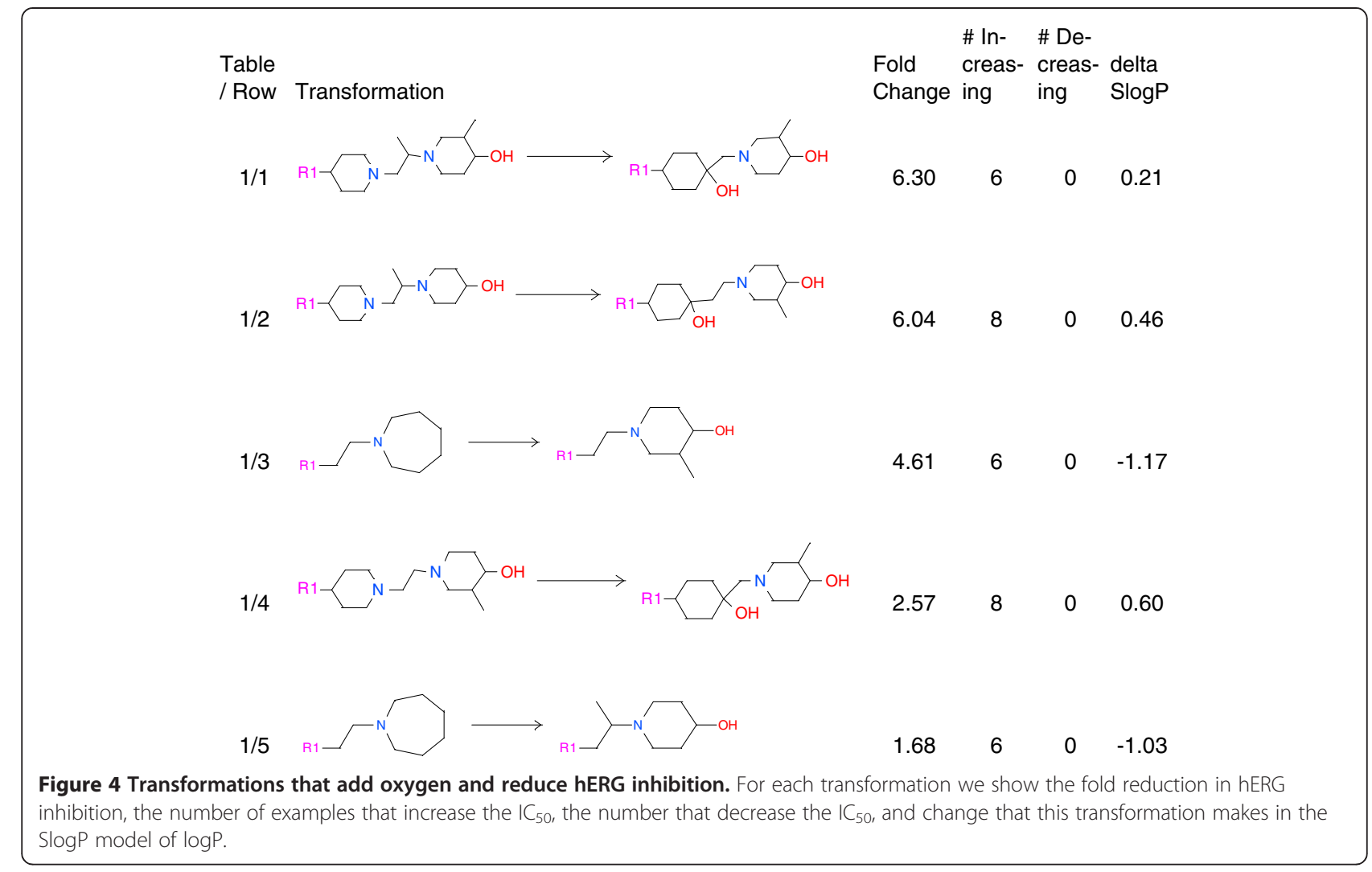

hERG blockers. While our observations reflect consistent behavior seen in the database, we cannot claim that they are completely general because of the narrowness of the molecular context. Due to their proprietary nature, we cannot disclose the full structures, but the molecules in the transformations are similar to each other. Given the range of changes in hERG inhibition $\mathrm{IC}_{50}$ s observed in our data set, improvements by 2 - and 3 -fold are notable.

Adding aliphatic oxygen frequently reduces hERG inhibition We observe that transformations that introduce a hydroxyl near an amine and changing a 7 member ring into a 6 membered ring and a methyl group reduce hERG inhibition by 2 to 6 fold (Figure 4, Rows 3 and 5). There are no transformations in which introducing a hydroxyl near an amine increases hERG binding (see Figure 4). Changing a molecule with two amines into a system with one amine and a hydroxyl group reduces hERG inhibition by 4 to 6 fold (Figure 4, Rows 1, 2 and 4), and increases SlogP/estimated lipophilicity.

\section{Aromatic substitutions reduce hERG inhibition}

Basic nitrogen atoms are the key to potent hERG blockers; however, introducing $s p 2$ nitrogens reduces inhibition in many transformations. Our largest observed reduction in hERG inhibition lowers hERG inhibition by 15 fold by adding $s p 2$ nitrogens to the slightly basic imidazole to obtain the somewhat less lipophilic methyl tetrazole (Figure 5, row 1). Changing a pyridyl nitrile to a $-\mathrm{CF} 3$ group reduces inhibition by 4.7 fold (Figure 5, row 2). We speculate that these reductions in hERG inhibition come from reducing lipophilicity and/or altering the energetics of pi-stacking of the inhibitor's aromatic groups with aromatic groups in the hERG channel. However, exploring this question is beyond the scope of this paper [16].

\section{Changing the environment of the amine nitrogen can reduce hERG inhibition}

Transformations in Figure 6 show that removing carbons and/or changing the electronic environment around the basic nitrogen can result in a modest reduction in hERG binding. The transformation in Figure 6, Row 1 shows us that removing carbon atoms and adding a hydroxyl has a consistent and substantial effect on hERG inhibition. Row 2 shows us that adding a cyclopropyl adjacent to nitrogen reduces the hERG inhibition. It is the reduction in basicity [17] that is likely responsible for this change. Three transformations show that removing carbon atoms reduces hERG inhibition (Figure 6, rows 3, 4 and 5). The last row (Figure 6, row 6) shows that the effect is not simply reducing lipophilicity, but is also from changing the chemical environment around the nitrogen, in particular the removal of the beta carbon. 


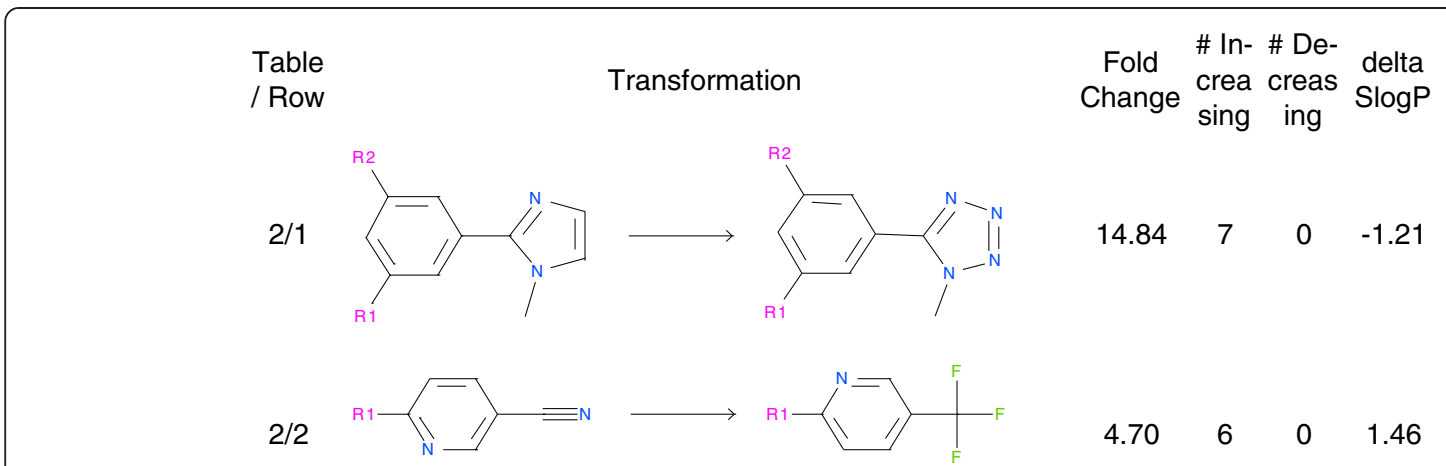

Figure 5 Transformations that alter an aromatic system. For both transformations we show the fold reduction in hERG inhibition, the number of examples that increase the $\mathrm{IC}_{50}$, the number that decrease the $\mathrm{IC}_{50}$, and change that this transformation makes in the Slog $\mathrm{P}$ model of $\log P$.

Miscellaneous transformations that reduce lipophilicity In this Figure the transformations that reduce hERG inhibition generally reduce lipophilicity. The transformation in Figure 7, row 1 which removes 2 carbons and changes the primary nitrogen into a secondary nitrogen improves hERG by 3 fold. Going from pyridyl, gem dimethyl hydroxyl to pyridyl nitrile (Figure 7, row 2) improves hERG by 2.5 fold and reduces inhibition in 7 out of 8 examples. Going from ethyl piperazine to methyl piperazine (Figure 7, row 3 ) results a 2.2 fold reduction in

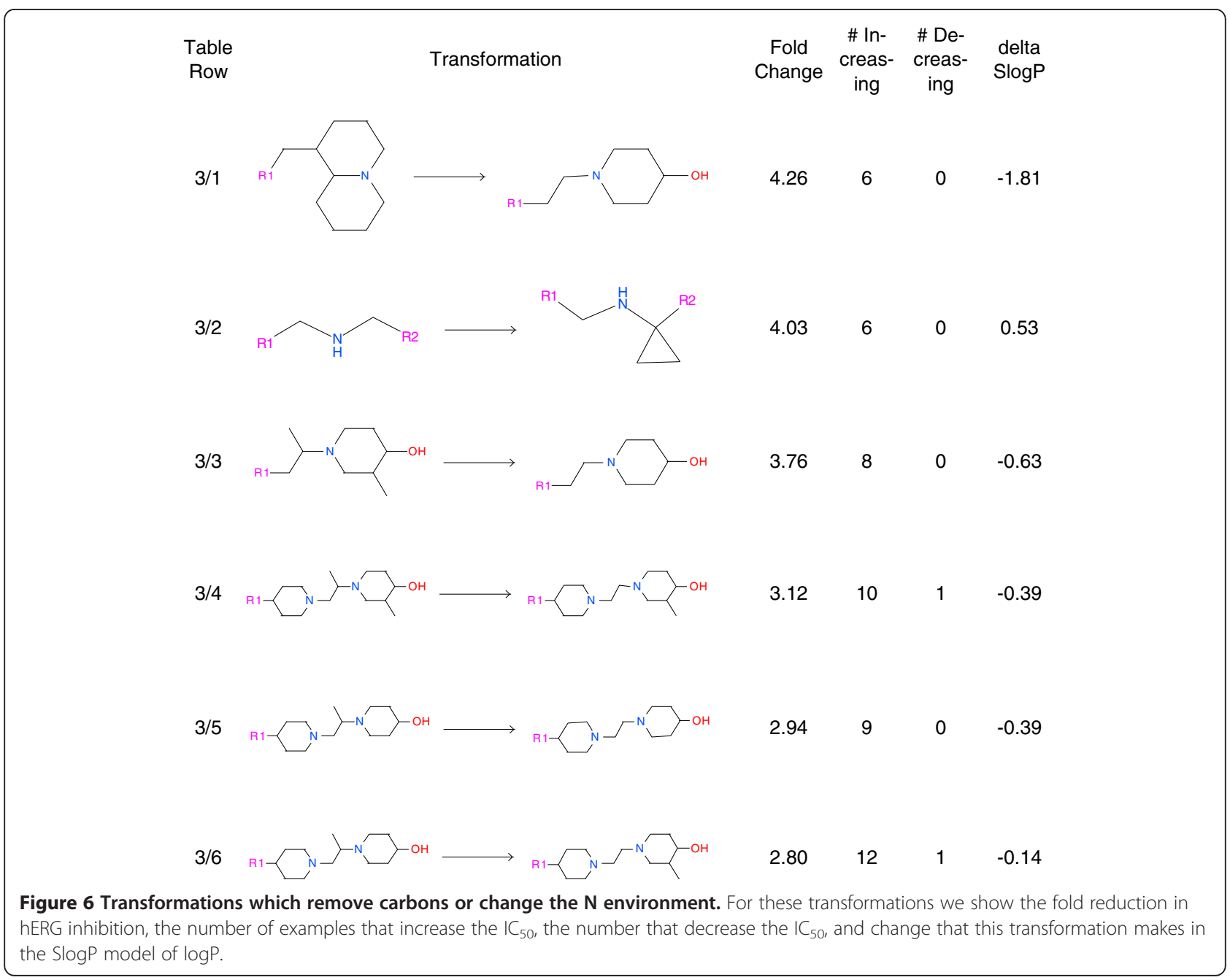


hERG inhibition. This change may result from lipophilicity and/or pKa effects on the nitrogen. A transformation from methyl to methoxy (Figure 7, row 4) has a small effect ( 1.7 fold), reducing hERG binding in 17 out of 21 examples.

\section{A comment on transformations not observed}

The direct addition of a carboxylic acid or the removal of an amine group does not occur in any aggregate with 5 or more examples. While we would expect these transformations to make a difference in hERG inhibition, our approach also looks at context, and none of these transformations appear with enough examples to pass our statistical threshold. Other techniques are needed to observe this effect (and others) in the data.

\section{$\Delta$ SlogP versus $\Delta \log (\mathrm{hERG}$ ) for the transformations}

Although we expect SlogP to make a difference in hERG inhibition, Figure 8 shows that its effect is not determinative. It is likely confounded by ionization and as well as other factors. We observe a number of transformations that reduce hERG inhibition even while increasing the SlogP. The points in Figure 8 are labeled with the Figure and Row in which they appear.

\section{Conclusions}

An attractive aspect of our approach, which does not use a preselected list of transformations, is that the resulting pair list is comprehensive in the data set used; that is, every transformation in the dataset is considered in the

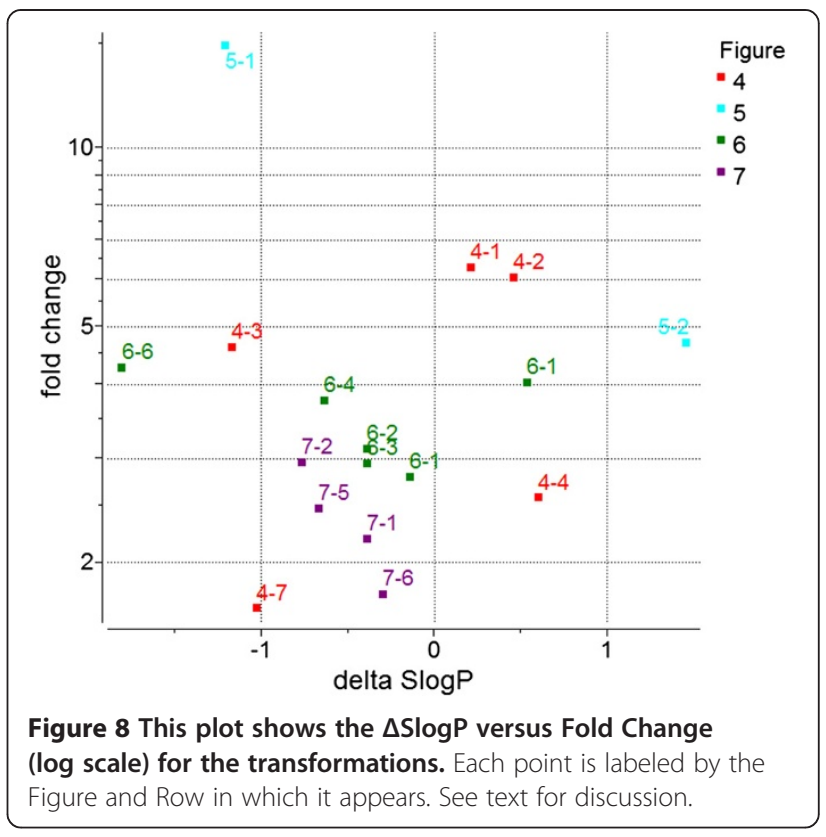

analysis. However because of the stringent statistical approach we applied most transformations are not used. One approach to extracting additional information from this dataset is to build a QSAR model on the vector fingerprint change of the transformations. Another attractive aspect of pair analysis for informing lead optimization is that the analysis is directly in the form of a change in chemical structure. Specific structural changes are revealed to reduce hERG binding, rather than indirect through

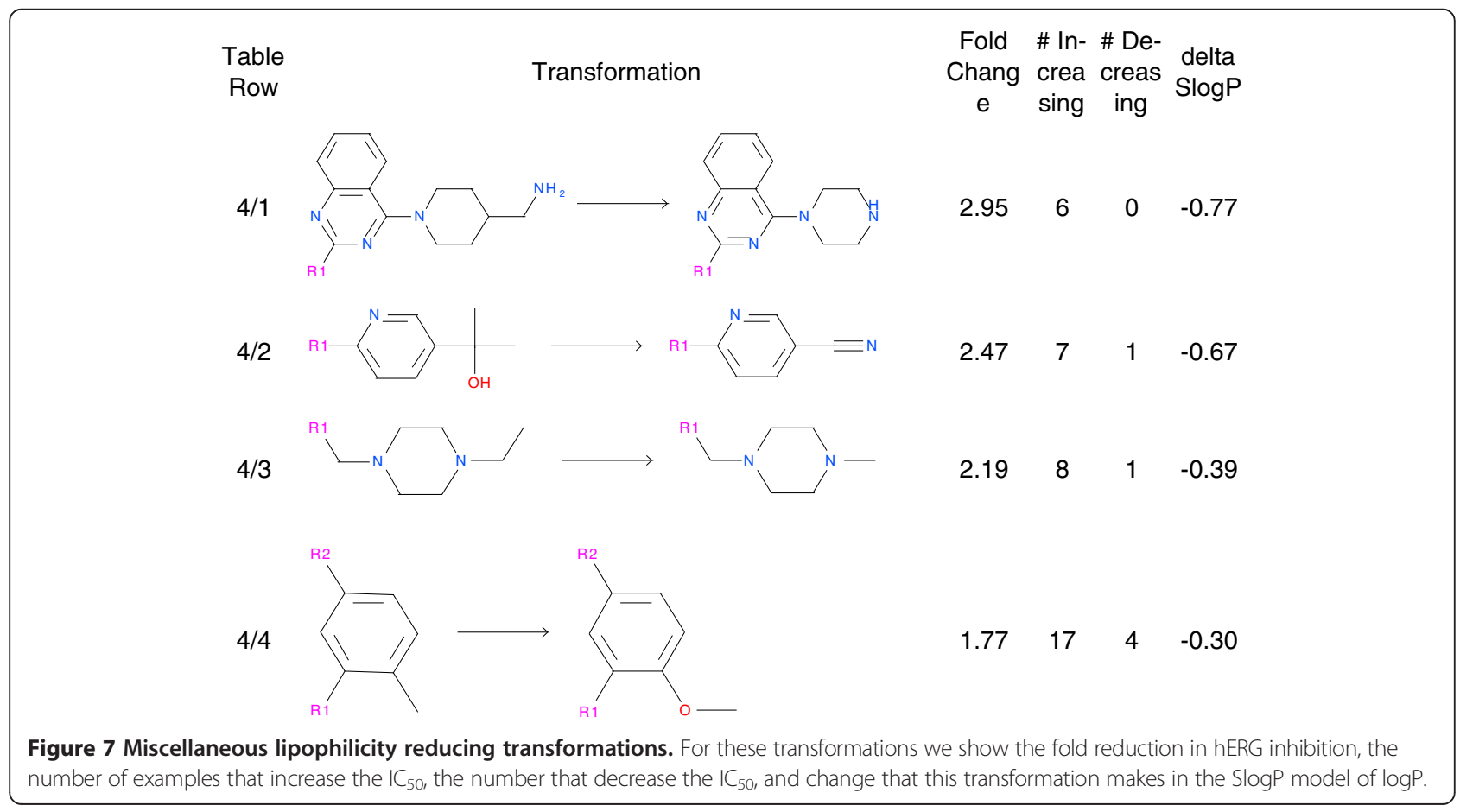


descriptors like SlogP [18], PSA [19], Chi-Square [20] or BCUT [21]. In broad terms, the transformations' effects are straightforward; for example, the removal of the basic nitrogen or the manipulation of the pKa of the basic nitrogen environment by addition or removal of nearby alkyl groups. This approach found some subtle transformations that reduce hERG binding, including adding hydroxyls, adding $s p 2$ nitrogens, or putting amide substitutions on aromatic rings. We expect that just as cheminformatics tools are currently applied to molecular representations to cluster, search and model molecules, these approaches can be applied to cluster, search and model transformations.

We observe that most transformations have no more than a modest change in hERG inhibition. This reinforces our impression from medicinal chemistry that hERG inhibition has a rather flat structure activity relationship: we rarely observe subtle changes in structure that result in dramatic changes in activity. Contrast this with biochemical potency, where subtle structural changes can often result in abolishing activity. Biochemical potency depends on receptors that have been designed by evolution to be sensitive to subtle changes in chemical structure (for example estrogen vs. testosterone, or epinephrine vs. norepinephrine) whereas the hERG channel has been designed to transport potassium and has had little or no evolutionary pressure to be selective against micromolar concentrations of aromatic amines.

\section{Methods}

Dofetilide displacement measurement data [22] were extracted from the Novartis corporate database. We computed the pairwise difference in the log of measured $\mathrm{IC}_{50}$ for each compound pair. Pairs were excluded if both measurements were off scale in the same direction. Otherwise, off-scale measurements were treated numerically as being at the extreme end of the scale; i.e., every value $>30 \mu \mathrm{M}$ was treated as $30 \mu \mathrm{M}$. We characterized the chemical structure with Pipeline Pilot's extended connectivity fingerprints (ECFP) [23]. We use the ECFP family of fingerprints because of their utility in cheminformatics applications [12,24]. The transformation between molecules was represented by the "difference fingerprint". In the difference fingerprint we record those ECFPs that disappear from the initial molecule and that appear in the final molecule (see Figure 1). The fingerprint approach is both much faster than maximum common substructure (MCS) methods and implicitly includes some molecular context. However, advances in MCS methods have reduced the computational effort needed to calculate MCS based matched pairs [25].

We define the aggregates by the change in fingerprint: pairs are put together in the same transformation if and only if they have the same change in fingerprint. In ECFP-N fingerprints, the molecules are characterized by substructures around each atom, and the ' $N$ ' denotes the maximum diameter of the substructures used. Thus, in ECFP0 fingerprints these substructures are just a count of the different atoms and the fingerprint has equivalent information to the molecular formula. We did not choose $\mathrm{N}=0$ because all transformations with the same change in molecular formula would have been aggregated together, and an aggregate would contain molecular pairs that are making different chemical transformations. Using ECFP2 has similar drawbacks. We initially tried $\mathrm{N}=4$, but not all the pairs it grouped together were similar enough. After tightening our criteria one step further to $\mathrm{N}=6$, the aggregates represent the same chemical transformation. However this came at the cost of spreading the available data over more aggregates, which reduced the number of aggregates with enough examples to make statistically definitive statements.

To assess the statistical power of a particular transformation, we consider the probability that a particular distribution of either increases or decreases $\mathrm{IC}_{50}$ would be observed by chance alone. Our null hypothesis is that the transformation on average has no systematic effect. From the Wilcoxon, we estimate the likelihood of observing a particular distribution $\mathrm{IC}_{50} \mathrm{~s}$ occurring by chance. In the null hypothesis, a sample size of 5 pairs which all either increase or decrease occurs $6.25 \%$ of the time. This gives us our threshold of 5 examples. For a sample size of 8 pairs, samples that have 7 increases and 1 decrease or 1 decrease and 7 increases occur (that is, has a p-value of) $5.46 \%$ of the time (see Additional file 1: Figure S1 on the paired Wilcoxon distribution).

Many biochemical assays have substantial correlation between measured $\mathrm{IC}_{50}$ and $\log \mathrm{P}$ (a measure of lipophilicity). Because we lack $\log \mathrm{P}$ measurements for many of our molecules we use a Crippen's model of $\log \mathrm{P}$. In particular, we use MOE's [26] (Chemical Computing Group, Montreal QC, Canada) implementation of that model which it calls SlogP [18]. SlogP is based on atom types, so each molecular pair in a particular aggregated transformation has the same change in SlogP.

\section{Additional file}

Additional file 1: Figure S1. Shows the Wilcoxon statistical significance for the different aggregiates observed in our data set. Each symbol in the graph represents an aggregate. The $x$-axis shows the number of examples that increase the hERG inhibition. The $y$-axis shows the number of examples that decrease hERG inhibition. The aggregate is colored by its Wilicoxon p-value (all the aggregates with the same number of increasing and deceasing examples have the sample Wilcoxon p-value). The total number of paired values is given by the sum of these two thus there is no 0,0 point. For example an aggregate with 0 increases, 5 decreases (that is 5 total) has significance value of $<0.05$ ( $~ 0.03)$. For an aggregate of 8 pairs, 1 increase and 7 decreases has statistical significance. 


\begin{abstract}
Abbreviations
$C_{\text {max }}$ : Maximum in vivo plasma concentration; SALI: Structure-activity landscape index; logP: Measurement of a compound's equilibrium partitioning between octanol and water; SlogP: LogP calculator found in $\mathrm{MOE}^{21}$; hERG: Human ether-a-go-go related gene; $\mathrm{IC}_{50}$ : Half maximal inhibitory concentration; MOE: Molecular operating environment, a software product of the Chemical Computing Group; MCS: Maximum common substructure; ECFP: Extended connectivity fingerprint; $\mu \mathrm{M}$ : Micromolar; nM: Nanomolar.
\end{abstract}

\section{Competing interests}

We have no competing interest financial or otherwise in these techniques.

\section{Authors' contributions}

CS devised the research plan, supervised the work, and drafted the manuscript. KS carried out the day-to-day computational work. Both authors read and approved the final manuscript.

\section{Authors' information}

CS works as CADD scientist at Novartis. KS was a summer intern in his lab.

\section{Acknowledgments}

We thank William Egan for valuable discussions. CS thanks Laszlo Urban, Paula Petrone, Patrick McCarren, and Andrew Rusinko for editorial help with the manuscript. The data used for this analysis was generated in the Lab of Steven Whitebread by Karl Chin, Alex Fekete, Fenghua Liu and Emily Nicholson. Peter Hunt provided us with initial Pipeline Pilot script. Also CS thanks John H. van Drie for introducing him to the cliff pair concept.

Received: 20 June 2013 Accepted: 10 September 2013

Published: 21 October 2013

\section{References}

1. Sanguinetti MC, Jiang C, Curran ME, Keating MT: A mechanistic link between an inherited and an acquired cardiac arrthytmia: HERG encodes the IKr potassium channel. Cell 1995, 81(2):299-307.

2. Redfern WS, Wakefield ID, Prior H, Pollard CE, Hammond TG, Valentin JP. Safety pharmacology - a progressive approach. Fundam Clin Pharmacol 2002, 16(3):161-173.

3. Roy M: HERG, a primary human ventricular target of the nonsedating antihistamine terfenadine. Circulation 1996, 94(4):817.

4. Whitebread S, Hamon J, Bojanic D, Urban L: Keynote review: in vitro safety pharmacology profiling: an essential tool for successful drug development. Drug Discov today 2005, 10(21):1421-1433.

5. Duda RO, Hart PE, Stork DG: Pattern classification. Hoboken NJ: WileyInterscience; 2000

6. Hastie T, Tibshirani R, Friedman J: The Elements of Statistical Learning: data mining, inference, and prediction. 2nd edition. Berlin: Springer-Verlag; 2009.

7. Keserü GM: Prediction of hERG potassium channel affinity by traditional and hologram QSAR methods. Bioorg Med Chem Lett 2003, 13:2773-2775.

8. Seierstad M, Agrafiotis D: A QSAR model of hERG binding using a large, diverse, and internally consistent training Set. Chem Biol Drug Des 2006, 67(4):284-296.

9. Maggiora GM: On outliers and activity cliffs - Why QSAR often disappoints. J Chem Inf Model 2006, 46(4):1535.

10. Guha R, Van Drie JH: Structure-activity landscape index: identifying and quantifying activity cliffs. J Chem Inf Model 2008, 48(3):646-658.

11. Van Drie $J H$, Guha R: Assessing how well a modeling protocol captures a structure activity landscape. J Chem Inf Model 2008, 48(8):1716-1728.

12. Sheridan RP, Hunt P, Culberson JC: Molecular transformations as a way of finding and exploiting consistent local QSAR. J Chem Inf Model 2006, 46(1):180-192.

13. Jamieson C, Moir EM, Rankovic Z, Wishart G: Medicinal chemistry of hERG optimizations: highlights and hang-Ups. J Med Chem 2006, 49(17):5029-5046.

14. Leach AG, Jones HD, Cosgrove DA, Kenny PW, Ruston L, MacFaul P, Wood JM, Colclough N, Law B: Matched molecular pairs as a guide in the optimization of pharmaceutical properties; a study of aqueous solubility, plasma protein binding and oral exposure. J Med Chem 2006, 49(23):6672-6682.

15. Papadatos $G$, Alkarouri M, Gillet VJ, Willett $P$, Kadirkamanathan V, Luscombe CN Bravi G, Richmond NJ, Pickett SD, Hussain J, Pritchard JM, Cooper AWJ,
Macdonald SJF: Lead optimization using matched molecular pairs: inclusion of contextual information for enhanced prediction of hERG inhibition, solubility, and lipophilicity. J Chem Inf Model 2010, 50(10):1872-1886.

16. Grimme S: Do special noncovalent $\pi-\pi$ stacking interactions really exist? Angew Chem 2008, 47(18):3430-3434.

17. Perrin CL, Fabian MA, Rivero I: Basicities of cycloalkylamines: baeyer strain theory revisited. Tetrahedron 2012, 55:5773-5780.

18. Wildman SA, Crippen GM: Prediction of physicochemical parameters by atomic contributions. J Chem Inf Comput Sci 1999, 39(5):868-873.

19. Ertl P: Fast calculation of molecular polar surface area as a Sum of fragment-based contributions and its application to the prediction of drug transport properties. J Med Chem 2000, 43:3714-3717.

20. Lipkowitz KB, Boyd DB, Hall LH, Kier LB: "The molecular connectivity Chi indexes and kappa shape indexes in structure property modeling" in Reviews. Comput Chem 1991, 2:367-422.

21. Pearlman RS, Smith KM: Novel software tools for chemical diversity. In 3D QSAR in drug design, 2. Edited by Kubinyi $\mathrm{H}$, Folkers G, Martin YC. Dordrecht: Kluwer Academic Publishers; 2002.

22. Finlayson K, Turnbull L, January CT, Sharkey J, Kelly JS: [3H]Dofetilide binding to HERG transfected membranes: a potential high throughput preclinical screen. Eur J Pharmacol 2001, 430(1):147-148.

23. Rogers D, Hahn M: Extended-connectivity fingerprints. J Chem Inf Model 2010, 50(5):742-754.

24. Glick M: Comparison of 2D-based descriptors for virtual screening using multiple bioactive reference structures. In Chemogenomics: an emerging strategy for rapid target and drug discovery. ; 2006:133-156.

25. Hussain J, Rea C: Computationally efficient algorithm to identify matched molecular pairs (MMPs) in large data sets. J Chem Inf Model 2010, 50(3):339-348.

26. Chemical Computing Group Inc: MOE (the molecular operating environment) version 2009.10. Montreal, Canada: Chemical Computing Group; 2012.

\section{doi:10.1186/1752-153X-7-167}

Cite this article as: Springer and Sokolnicki: A fingerprint pair analysis of hERG inhibition data. Chemistry Central Journal 2013 7:167.

Publish with ChemistryCentral and every
scientist can read your work free of charge
"Open access provides opportunities to our
colleagues in other parts of the globe, by allowing
anyone to view the content free of charge."
W. Jeffery Hurst, The Hershey Company.
- available free of charge to the entire scientific community
- peer reviewed and published immediately upon acceptance
- cited in PubMed and archived on PubMed Central
- yours - you keep the copyright
submit your manuscript here:
http://www.chemistrycentral.com/manuscript/

